\title{
The catechol-O-methyltransferase (COMT) Val158Met genotype modulates working memory-related dorsolateral prefrontal response and performance in bipolar disorder
}

\author{
KW Miskowiak ${ }^{1,2}$ (D) | HL Kjærstad ${ }^{1}$ (D) | MM Støttrup ${ }^{1}$ | AM Svendsen ${ }^{3}$ | \\ KM Demant $^{1}$ | LK Hoeffding,5,6 | TM Werge ${ }^{4,5,7}$ | KE Burdick ${ }^{8}$ | KDomschke ${ }^{9}$ |

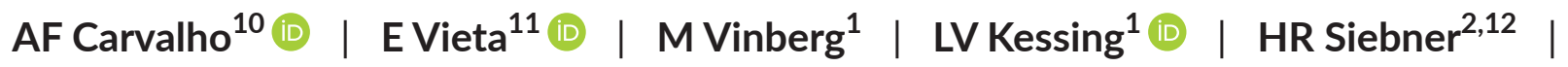 \\ J Macoveanu $^{1,2}$ \\ ${ }^{1}$ Copenhagen Affective Disorder Research Centre, Psychiatric Centre Copenhagen, Copenhagen University Hospital, Rigshospitalet, Denmark \\ ${ }^{2}$ Danish Research Centre for Magnetic Resonance, Centre for Functional and Diagnostic Imaging and Research, Hvidovre Hospital, University of Copenhagen, \\ Copenhagen, Denmark \\ ${ }^{3}$ Mental Health Centre Copenhagen, Capital Region of Denmark, Copenhagen, Denmark \\ ${ }^{4}$ Institute of Biological Psychiatry, MHC Sct. Hans, Mental Health Services, Copenhagen, Denmark \\ ${ }^{5}$ The Lundbeck Foundation Initiative for Integrative Psychiatric Research, iPSYCH, Aarhus, Denmark \\ ${ }^{6}$ Department of Clinical Immunology, University Hospital of Copenhagen Rigshospitalet, Copenhagen, Denmark \\ ${ }^{7}$ Department of Clinical Medicine, University of Copenhagen, Copenhagen, Denmark \\ ${ }^{8}$ Brigham and Women's Hospital, Harvard Medical School, Boston, MA, NY, USA \\ ${ }^{9}$ Department of Psychiatry, University of Freiburg, Freiburg, Germany \\ ${ }^{10}$ Translational Psychiatry Research Group and Department of Clinical Medicine, Federal University of Ceará, Fortaleza, Brazil \\ ${ }^{11}$ Clinical Institute of Neuroscience, Hospital Clinic, University of Barcelona, IDIBAPS, CIBERSAM, Barcelona, Catalonia, Spain \\ ${ }^{12}$ Department of Neurology, Copenhagen University Hospital, Bispebjerg, Denmark
}

\section{Correspondence}

Kamilla Woznica Miskowiak, CADIC,

Psychiatric Centre Copenhagen, Copenhagen

University Hospital, Rigshospitalet,

Copenhagen, Denmark.

Email: Kamilla@miskowiak.dk

Funding information

Lundbeck Foundation, Grant/Award Number:

R219-2016-1030; TrygFonden; Danish

Council for Independent Research; Novo Nordisk Foundation; Grant/Award Number: R144-A11605; Beckett Fonden; Grant/Award Number: 525 PV/LS; Savværksejer Juhl's Mindefond; Danish Council for Independent Research; Grant/Award Number: 271-08-0937
Objectives: Cognitive dysfunction affects a substantial proportion of patients with bipolar disorder (BD), and genetic-imaging paradigms may aid in the elucidation of mechanisms implicated in this symptomatic domain. The Val allele of the functional Val158Met polymorphism of the catechol-O-methyltransferase (COMT) gene is associated with reduced prefrontal cortex dopamine and exaggerated working memoryrelated prefrontal activity. This functional magnetic resonance imaging (fMRI) study investigated for the first time whether the COMT Val158Met genotype modulates prefrontal activity during spatial working memory in BD.

Methods: Sixty-four outpatients with BD in full or partial remission were stratified according to COMT Val158Met genotype (ValVal [n=13], ValMet [n=34], and MetMet $[n=17])$. The patients completed a spatial $n$-back working memory task during fMRI and the Cambridge Neuropsychological Test Automated Battery (CANTAB) Spatial Working Memory test outside the scanner.

Results: During high working memory load (2-back vs 1-back), Val homozygotes displayed decreased activity relative to ValMet individuals, with Met homozygotes displaying intermediate levels of activity in the right dorsolateral prefrontal cortex (dIPFC) $(P=.016)$. Exploratory whole-brain analysis revealed a bilateral decrease in working 
memory-related dIPFC activity in the ValVal group vs the ValMet group which was not associated with differences in working memory performance during fMRI. Outside the MRI scanner, Val carriers performed worse in the CANTAB Spatial Working Memory task than Met homozygotes $(P \leq .006)$, with deficits being most pronounced in Val homozygotes.

Conclusions: The association between Val allelic load, dIPFC activity and WM impairment points to a putative role of aberrant PFC dopamine tonus in the cognitive impairments in BD.

\section{KEYWORDS}

bipolar disorder, catechol-O-methyltransferase (COMT) Val158Met, functional magnetic resonance imaging (fMRI), working memory

\section{INTRODUCTION}

Cognitive dysfunction across attention, memory, and executive function is a core feature of bipolar disorder (BD), which persists after clinical remission from mood episodes and impedes patients' functional recovery. ${ }^{1,2}$ Unaffected first-degree relatives of patients with BD display similar non-specific, albeit generally milder, cognitive deficits, ${ }^{3}$ indicating that cognitive dysfunction is associated with genetic liability for BD. Functional magnetic resonance imaging (fMRI) studies of $\mathrm{BD}$ patients and high-risk groups show consistently that the cognitive deficits are accompanied by aberrant (hypo- and hyper-) activity in the dorsolateral and medial prefrontal cortex (dIPFC and mPFC) during working memory (WM) performance. ${ }^{4-11}$ Nevertheless, there is little understanding of the association between cognitive deficits, abnormal neural activity, and genetic liability for BD.

A risk gene encoding the enzyme catechol-O-methyltransferase (COMT) has attracted attention as a common candidate gene for both neuropsychiatric illness and cognitive dysfunction. ${ }^{12}$ The functional Val158Met polymorphism (rs4680) of the COMT gene mediates the degradation of dopamine (DA) in the synaptic cleft, and its Val allele seems to be associated with lower levels of synaptic DA in the PFC, while the Met allele is related to higher PFC DA levels. ${ }^{13}$ Evidence from animal and human studies suggests that hyper- and hypodopaminergic states contribute to mania and depression, respectively (eg, 14,15). Thus, an increase of synaptic DA levels can create manic phenotypes (eg, 16). Conversely, psychotropics that cause DA depletion can induce a depressed state (eg, 17,18), whereas the DA agonist pramipexole attenuates depressive symptoms in depressed $B D$ patients. ${ }^{19-21}$ The relationship between DA activity in the PFC and cognitive performance has been described as an inverted U-shape, in which both high and low cortical DA tonus is associated with cognitive impairments. ${ }^{22}$ Indeed, there is relatively consistent evidence from studies on schizophrenia and healthy samples for poorer cognitive function in Val homozygotes (with low PFC DA tonus) relative to Met carriers, particularly within the executive function domain, ${ }^{12,23-26}$ although some studies found no such association. ${ }^{27-29}$ At the neural level, the Val allele (particularly Val homozygosity) is associated with exaggerated dIPFC activity during n-back WM across patients with schizophrenia and healthy individuals where no behavioral impairments are observed. ${ }^{13,30-35}$ This evidence has led to the hypothesis of inefficient prefrontal processing in Val homozygotes, as reflected by a need to recruit greater dIPFC resources to maintain normal task performance. $^{36,37}$

Despite evidence linking the COMTVal158Met genotype to neural and cognitive impairments, its influence on neurocognitive function in $\mathrm{BD}$ is unclear. One study found that cognitive dysfunction in patients with BD type I is modulated by the presence of a Val allele, with Val carriers $(n=54)$ exhibiting lower deficits in executive function and verbal fluency relative to Met homozygotes $(n=18)$ during manic and mixed episodes. ${ }^{38}$ Another study found no effect of the Val allele on attention, verbal memory, or executive function in 52 clinically stable patients with BD type $1,{ }^{39}$ although a second functional polymorphism within COMT (rs165599) was found to influence verbal memory. However, no neuroimaging study has investigated the association between COMT Val158Met and WM-related neural response in $\mathrm{BD}$, which could provide a more sensitive assay of abnormal brain function than behavioral measures. ${ }^{40,41}$ The present fMRI study therefore aimed to investigate whether the COMT Val158Met polymorphism modulates dIPFC activity during the spatial n-back WM task and WM performance in remitted patients with BD. We hypothesized that Val homozygous remitted BD patients would exhibit (i) greater dIPFC activity during high-load n-back WM and (ii) impaired performance on a spatial WM test relative to Met carriers.

\section{2 | METHODS}

\section{1 | Study design and participants}

The study included baseline data from patients with BD recruited for two randomized intervention studies targeting cognitive dysfunction. ${ }^{42,43}$ Patients, $18-65$ years of age, were screened with Schedules for Clinical Assessment in Neuropsychiatry to confirm ICD-10 diagnosis of BD and were rated with the Hamilton Depression Rating Scale (HDRS-17; REF) and Young Mania Rating Scale (YMRS) to confirm 
partial or full remission (HDRS-17 and YMRS scores <14). Participants reported moderate to severe cognitive difficulties according to the Cognitive and Physical Functioning Questionnaire (CPFQ) ${ }^{44}$ (score $\geq 4$ on at least two domains). The original studies were approved by the local ethics committee, Danish Medicines Agency, and Danish Data Agency, and registered at clinicaltrials.gov (numbers NCT00916552 and NCT01457235, respectively). After complete description of the study, written informed consent was obtained from all participants. For further details on the recruitment and screening procedures, please refer to previous work. ${ }^{42,43}$

\section{2 | Genotyping}

Genomic DNA was extracted from blood samples using the Maxwell Blood DNA purification kit (Promega, Madison, WI, USA) in accordance with the manufacturer's protocol and genotyped using the Illumina Infinium PsychArrayBeadChip (Illumina, San Diego, CA, USA).

\section{3 | Neurocognitive tests}

$\mathrm{N}$-back WM tasks were employed as they are widely used to probe memory aspects of executive function. ${ }^{45}$ During fMRI, participants performed a spatial $n$-back WM task. ${ }^{46}$ The task consisted of blocks of three types of conditions that differed in terms of WM load (0-back, 1-back and 2-back). In each condition, a yellow circle appeared in a sequence of 14 appearances at random locations in a 5 by 5 grid. The circle was displayed for 300 ms followed by an empty grid for 1200 ms. In 1-back and 2-back conditions, patients indicated with a button press when the circle appeared at the same location as one trial and two trials back, respectively. During the 0 -back condition, patients pressed a button when the circle appeared in one of the four grid corners. The blocks had an average of three target trials and were presented successively five times ( 15 blocks in total) interleaved with 8-second fixation crosses. The total task length was 7 minutes 35 seconds.

Within the week of the fMRI scan [mean \pm standard deviation (SD) $3.3 \pm 2.5$ days], participants were also given a spatial working memory (SWM) task from the Cambridge Neuropsychological Test Automated Battery (CANTAB) on a laptop computer as part of a larger neurocognitive test battery (for details see Demant et al. ${ }^{47}$ ). This task was selected for the present study because it probes similar aspects of WM to the spatial n-back WM paradigm administered inside the scanner, thus providing a sensitive behavioral read-out of spatial WM. Moreover, we were interested in the domain of WM more specifically due to prior reported effects of COMT on this domain. Behavioral outcome measures of interest were the 'between errors' score, as indicated by the number of errors made (i.e., on 4-8 boxes), and the 'strategy' score, specifying the number of times inefficient search strategies were used.

\section{4 | Magnetic resonance imaging}

Whole-brain MRI data were collected at the Danish Research Centre for Magnetic Resonance with a 3 Tesla Siemens Trio MR scanner (Siemens, Erlangen, Germany) using an eight-channel head array coil.
Blood-oxygen-level-dependent (BOLD)-sensitive fMRI used a T2* weighted echo-planar imaging (EPI) sequence with an echo time (TE) of $30 \mathrm{~ms}$, a repetition time (TR) of $2.49 \mathrm{~ms}$ and a low flip angle of $20^{\circ}$ to minimize physiological noise. ${ }^{48}$ For the WM task, a total of 184 brain volumes were acquired in a single $\mathrm{fMRI}$ session, each consisting of 42 slices with a slice thickness of $3 \mathrm{~mm}$ and a field of view (FOV) of $192 \times 192 \mathrm{~mm}$ using a $64 \times 64$ grid. High-resolution 3D structural T1-weighted spin echo images were obtained after the first session of BOLD fMRI (TI=800, TE=3.93, TR=1540 ms, flip angle $9^{\circ} ; 256 \times 256$ FOV; 192 slices).

\section{5 | fMRI data analysis}

Functional MRI data processing was performed with the FMRI Expert Analysis Tool (FEAT; version 6.00), part of FMRIB's Software Library (FSL; www.fmrib.ox.ac.uk/fsl). Pre-processing included image realignment, non-brain removal, spatial normalization to a Montreal Neurologic Institute (MNI) template and spatially smoothing (Gaussian kernel, $5 \mathrm{~mm}$ full-width-half-maximum). The time series in each session were high pass-filtered (to $\max 0.008 \mathrm{~Hz}$ ). Three conditions, '1back', '2-back' and '0-back', were modeled as blocks convolved with a canonical hemodynamic response function.

To investigate the hypothesis that the COMT Val158Met genotype would modulate spatial WM-relevant activity in the PFC, we defined a spherical region of interest (ROI) with a $10 \mathrm{~mm}$ radius around the peak of the right dIPFC ( $x=40, y=34, z=29)$ involved in spatial WM processes as reported in a meta-analysis. ${ }^{45}$ The ROI was constructed on the MNI template using the WFU PickAtlas toolbox. ${ }^{49}$ We extracted the mean percent BOLD signal change in the dIPFC ROI during 1-, 2- and 0-back and computed the differential signal change for the 2-back>0-back and 2-back>1-back contrasts to assess the effects of COMT genotype on neural response during general WM and high-load specific WM, respectively. Analysis of the extracted mean percent signal change in the dIPFC ROI was performed in Statistical Package for Social Sciences (SPSS; version 22; IBM Corporation, Armonk, NY, USA) using two one-way ANOVA models with COMT genotype (ValVal, ValMet and MetMet) as an independent factor and high-load specific WM (2-back>1-back) and general WM (2-back>0-back) as dependent factors, respectively. Bonferroni correction was applied to adjust for the number of comparisons of signal change from the dIPFC ROI (adjusted threshold for significance: $P=.05 / 2=.025$ ).

We also carried out a whole-brain exploratory analysis. Individual subject data were computed using the general linear model with local autocorrelation correction. ${ }^{50}$ The individual contrasts of interest (2-back>1-back or 2-back>0-back) were included in separate general linear models with nonparametric permutation inference $(n=5000)$ using the 'randomize' algorithm implemented in FSL. ${ }^{51}$ The main effect of COMT genotype was assessed using an $F$ test. Significant clusters were identified using the threshold-free cluster enhancement method at corrected $P<.05$. The mean percent BOLD signal change in clusters showing significant differences between groups was extracted for visualization purposes. A standard anatomical atlas ${ }^{52}$ was used to localize peak cluster activations. 


\subsection{Statistical analyses of behavioral and mood data}

For the spatial n-back WM task, signal detection theory was applied to obtain a measure of spatial n-back WM accuracy corrected for participants' response tendency $\left(d^{\prime}\right) .{ }^{53}$ Outliers, defined as WM accuracy of $\geq 2$ SD below the sample's mean, were excluded. The influence of COMT genotype on spatial n-back WM performance (accuracy and speed) during scanning was examined with $\mathrm{WM}$ load $^{1,2}$ as the within-subject factor and genotype (ValVal, ValMet or MetMet) as the between-subject factor (all tests were two-tailed). Significant interactions were followed up by independent-samples $t$ tests. For the SWM task, the effect of genotype on spatial WM performance (strategy and errors, respectively) was examined using one-way ANOVA with genotype as the between-subjects factor. Effects of COMT Val158Met genotype on these behavioral measures were subjected to Bonferroni corrections for multiple comparisons (adjusted thresholds for significance: $n$-back WM: $P=.05 / 4=0.0125$; SWM: $P=.05 / 2=.025)$. For any statistically significant interactions between genotype and cognitive performance/brain activity, we (i) conducted post hoc analysis of covariance (ANCOVA) with adjustment for any potential differences in demographic variables to explore their possible influence on the findings and (ii) explored the potential interacting effects of COMT genotype and gender on cognitive performance and dIPFC activity, given emerging evidence for influence of COMT genotype on gender differences in brain function. ${ }^{54}$ Exploratory correlational analyses were conducted between clusters showing differential effects of COMT
Val158Met genotype on WM-related activity and WM performance, mood symptoms and medication status. These post hoc analyses were not adjusted for multiple comparisons due to their exploratory nature. Finally, medication status across the genotype groups was examined with Pearson's chi square. Statistical analyses were performed in SPSS (version 22; IBM Corporation, Armonk, NY, USA).

\section{3 | RESULTS}

\section{1 | Patient characteristics}

Four individuals with WM accuracy of $\geq 2$ SD below the mean were excluded from the $\mathrm{FMRI}$ and behavioral analyses, resulting in a total sample of 64 participants. Genotype groups (ValVal: N=13; ValMet: $\mathrm{N}=34$; MetMet: $\mathrm{N}=17$ ) were comparable for age, gender, education level, mood, and medication ( $P>.05$; see Table 1 ). ValVal individuals were characterized by numerically older age and longer illness duration than Met carriers, although this was not statistically significant (Table 1). The allelic frequencies for the total sample were 0.47 for the $\mathrm{Val}$ allele and 0.53 for the Met allele. Genotype frequencies were confirmed to be in Hardy-Weinberg equilibrium ( $P=.59)$.

\subsection{Behavioral results}

The WM accuracy ( $d$ ') score data showed a negative skew due to near-ceiling accuracy rates. As $d^{\prime}$ scores are in the range of $0-1$, we arcsine transformed the data to approach a normal distribution prior
TABLE 1 Demographic and clinical data according to catechol-O-methyltransferase (COMT) Val158Met genotype

\begin{tabular}{|c|c|c|c|c|}
\hline & \multicolumn{3}{|c|}{ COMT Val158Met genotype } & \multirow[b]{2}{*}{$P$-value } \\
\hline & $\begin{array}{l}\text { ValVal } \\
(\mathrm{N}=13)\end{array}$ & $\begin{array}{l}\text { ValMet } \\
(\mathrm{N}=34)\end{array}$ & $\begin{array}{l}\text { MetMet } \\
(\mathrm{N}=17)\end{array}$ & \\
\hline Age, mean (SD) & $42(10)$ & $35(11)$ & $36(9)$ & 0.12 \\
\hline Gender, female, n (\%) & $9(69)$ & $19(56)$ & $10(59)$ & 0.71 \\
\hline Education, mean (SD) & $15(2)$ & $15(3)$ & $14(5)$ & 0.62 \\
\hline HDRS-17, mean (SD) & $9(5)$ & $7(5)$ & $8(4)$ & 0.62 \\
\hline YMRS, mean (SD) & $3(3)$ & $2(2)$ & $2(2)$ & 0.87 \\
\hline No. prior depressions, mean (SD) & $8(11)$ & $5(3)$ & $9(9)$ & 0.10 \\
\hline No. prior (hypo)manias, mean (SD) & $10(14)$ & $6(7)$ & $11(10)$ & 0.16 \\
\hline Age at debut (years) & $22(12)$ & $20(8)$ & $20(10)$ & 0.82 \\
\hline Illness duration (years) & $21(10)$ & $15(9)$ & $16(11)$ & 0.19 \\
\hline Bipolar disorder type II subtype, n (\%) & $5(39)$ & $15(44)$ & $8(47)$ & 0.90 \\
\hline \multicolumn{5}{|l|}{ Current medication } \\
\hline Lithium, n (\%) & $5(39)$ & $15(44)$ & $8(47)$ & 0.66 \\
\hline Anticonvulsants, n (\%) & $9(69)$ & $20(59)$ & $8(47)$ & 0.57 \\
\hline Antidepressants, n (\%) & $5(39)$ & $14(41)$ & $6(35)$ & 0.99 \\
\hline Antipsychotics, n (\%) & $7(54)$ & $9(27)$ & $5(29)$ & 0.14 \\
\hline Benzodiazepines, n (\%) & $4(31)$ & $9(27)$ & $2(12)$ & 0.51 \\
\hline Melatonin, n (\%) & $1(8)$ & $2(6)$ & $0(0)$ & 0.59 \\
\hline No medications, mean (SD) & $0(0)$ & $0(0)$ & $0(0)$ & 1.00 \\
\hline No. of medications, mean (SD) & $2.5(0.9)$ & $2.0(0.9)$ & $2.1(0.5)$ & 0.21 \\
\hline
\end{tabular}

HDRS-17, Hamilton Depression Rating Scale; standard deviation; YMRS, Young Mania Rating Scale. 
TABLE 2 n-Back working memory (WM) performance according to catechol-O-methyltransferase (COMT) Val158Met genotype

\begin{tabular}{|c|c|c|c|c|}
\hline \multicolumn{5}{|c|}{$\mathrm{N}$-back WM performance in the scanner } \\
\hline & Mean (SD) & $F$ & $d f$ & $P$-value \\
\hline \multicolumn{5}{|c|}{ Accuracy (arcsine transformed $d^{\prime}$ ' values) } \\
\hline \multicolumn{5}{|c|}{ WM across 1- and 2-back } \\
\hline COMT ValVal & $1.09(0.17)$ & 1.44 & 2,61 & .25 \\
\hline COMT ValMet & $1.12(0.17)$ & & & \\
\hline COMT MetMet & $1.18(0.09)$ & & & \\
\hline \multicolumn{5}{|l|}{ 1-back WM } \\
\hline COMT ValVal & $1.22(0.15)$ & 0.56 & 2,61 & .58 \\
\hline COMT ValMet & $1.25(0.19)$ & & & \\
\hline COMT MetMet & $1.29(0.12)$ & & & \\
\hline \multicolumn{5}{|l|}{ 2-back } \\
\hline COMT ValVal & $0.98(0.26)$ & 1.42 & 2,61 & .25 \\
\hline COMT ValMet & $1.02(0.22)$ & & & \\
\hline COMT MetMet & $1.09(0.10)$ & & & \\
\hline \multicolumn{5}{|c|}{ Response times (for correct detection) } \\
\hline \multicolumn{5}{|l|}{ 0-back control } \\
\hline COMT ValVal & $592(65)$ & 0.86 & 2,61 & .43 \\
\hline COMT ValMet & $554(98)$ & & & \\
\hline COMT MetMet & $559(92)$ & & & \\
\hline \multicolumn{5}{|l|}{ 1-back WM } \\
\hline COMT ValVal & $638(113)$ & 1.48 & 2,61 & .24 \\
\hline COMT ValMet & $575(102)$ & & & \\
\hline COMT MetMet & $588(115)$ & & & \\
\hline \multicolumn{5}{|l|}{ 2-back WM } \\
\hline COMT ValVal & $636(136)$ & 0.81 & 2,61 & .45 \\
\hline COMT ValMet & $595(125)$ & & & \\
\hline COMT MetMet & $576(129)$ & & & \\
\hline \multicolumn{5}{|l|}{ SWM outside scanner } \\
\hline \multicolumn{5}{|l|}{ SWM strategy } \\
\hline COMT ValVal & $35.3(6.7)$ & 5.65 & 2,56 & .006 \\
\hline COMT ValMet & $34.1(6.5)$ & & & \\
\hline COMT MetMet & $28.1(5.6)$ & & & \\
\hline \multicolumn{5}{|l|}{ SWM errors } \\
\hline COMT ValVal & $37.8(17.4)$ & 8.36 & 2,56 & .001 \\
\hline COMT ValMet & $27.2(20.7)$ & & & \\
\hline COMT MetMet & $10.1(11.2)$ & & & \\
\hline
\end{tabular}

SD, standard deviation; SWM, spatial working memory.

to statistical analysis. ${ }^{55}$ There were no differences between the three genotype groups in $n$-back WM accuracy $(P \geq .26)$ or speed $(P \geq .31)$ (Table 2). In contrast, performance on the CANTAB SWM test outside the scanner showed statistically significant differences between groups on SWM 'strategy' $\left(F_{2,56}=5.65, P=.006\right)$ and SWM 'errors' $\left(F_{2,56}=8.36, P=.001\right)$ (Figure 1$)$, which remained statistically significant after post hoc adjustment for age $(P \leq .009)$ and illness duration $(P \leq .006)$. These interactions were driven by less efficient strategies

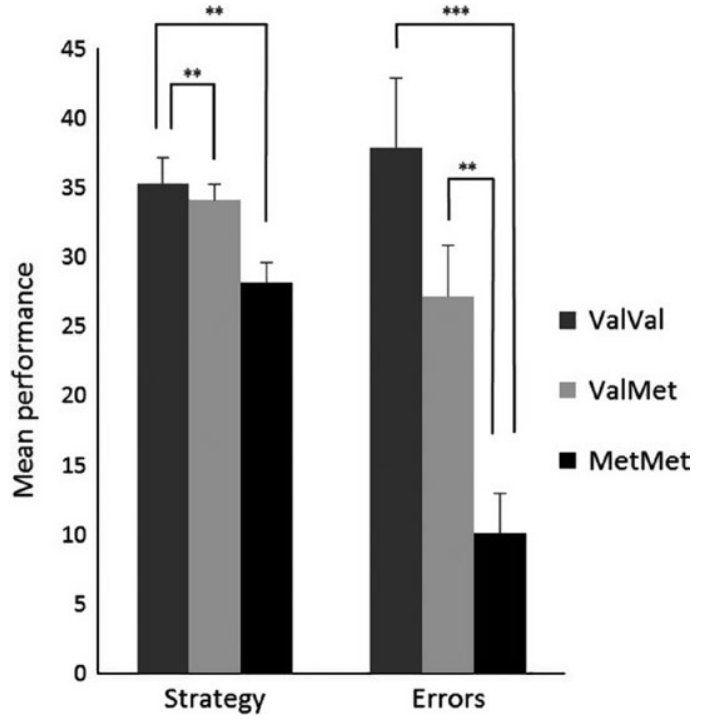

FIGURE 1 Mean performance (strategy and errors) during the Cambridge Neuropsychological Test Automated Battery (CANTAB) spatial working memory task outside the scanner stratified for catechol-O-methyltransferase (COMT) Val158Met genotype. Higher values indicate more inefficient strategies and more errors, respectively. Error bars represent the standard error. There were significant differences between groups on SWM 'strategy' $\left(F_{2,56}=5.65, P=.006\right)$ and SWM 'errors' $\left(F_{2,56}=8.36, P=.001\right)$. The interactions were driven by less efficient strategies and more errors in the Val carriers than Met homozygotes (strategy: ValVal vs MetMet: $t=3.03, d f=25, P=.006$; Cohen's $d=1.16$; ValMet vs MetMet: $t=3.05, d f=45, P=.004$; Cohen's $d=0.98$; errors: ValVal vs MetMet: $t=5.03, d f=25, P<.001$; Cohen's $d=1.90$; ValMet vs MetMet: $t=2.99$, $d f=45, \underline{P}=.005$; Cohen's $d=1.03$ ). ${ }^{* *}$ corresponds to a significance level of $P<0.01$; ${ }^{* * *}$ corresponds to a significance level of $P<0.001$.

and more errors in the Val carriers compared with Met homozygotes (strategy: ValVal vs MetMet: $t=3.03, d f=25, P=.006$; Cohen's $d=1.16$; ValMet vs MetMet: $t=3.05, d f=45, P=.004$; Cohen's $d=0.98$; whereas ValVal vs ValMet: $P>.61$; errors: ValVal vs MetMet: $t=5.03$, $d f=25, P<.001$; Cohen's $d=1.90$; ValMet vs MetMet: $t=2.99, d f=45$, $P=.005$; Cohen's $d=1.03$; whereas ValVal vs ValMet: $P>.12$ ). These findings are consistent with a linear trend based upon Val allelic load. No interacting effects of COMT genotype and gender were found on SWM strategy and errors, respectively $(P \geq 80)$.

\section{3 | fMRI results}

\subsection{1 | A priori dorsolateral prefrontal cortex region of interest}

The right dIPFC ROI was significantly activated during the WM task (2-back>0-back) ( $t=12.71, d f=63, P<.001)$ and showed greater response under high than low WM loads (2-back $>1$-back) $(t=6.19, d f=63$, $P<.001)$. Dorsolateral PFC response showed no differences between genotype groups during general WM (2-back $>0$-back) $(P>.54)$, but was differentially activated between the groups during high-load specific WM (2-back>1-back) $\left(F_{2,61}=4.45, P=.016\right)$. This interaction was driven 


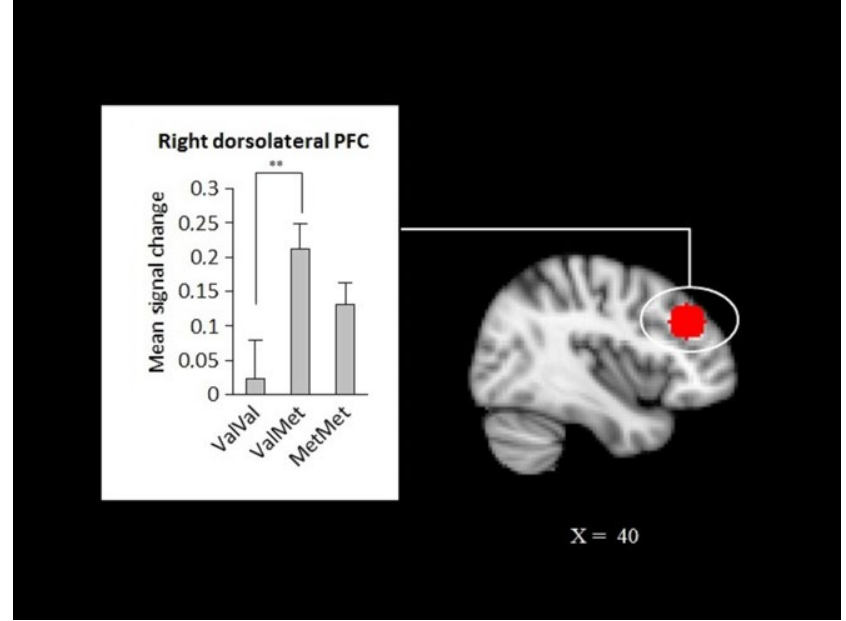

FIGURE 2 A priori region of interest (ROI) in the right dorsolateral prefrontal cortex (dIPFC) (red) and plot of mean blood-oxygen-leveldependent (BOLD) signal change during high working memory (WM) load (2-back>1-back) across the catechol-O-methyltransferase (COMT) Val158Met genotype groups. Bars show the mean; error bars show the standard error. The dIPFC showed differential response between the groups during high-load specific $W M\left(F_{2,61}=4.45, P=.016\right)$, which was driven by lower activity in ValVal compared to ValMet individuals ( $t=2.73, d f=45, P=.009$; Cohen's $d=0.90$ ) and a strong trend towards lower response in ValVal than MetMet individuals $(t=2.03, d f=19.32$, $P=.06$ ). ${ }^{* *}$ corresponds to a significance level of $P<0.01$. [Colour figure can be viewed at wileyonlinelibrary.com]

by lower activity in ValVal compared to ValMet individuals ( $t=2.73$, $d f=45, P=.009$; Cohen's $d=0.90$ ) and a strong trend towards lower response in ValVal than MetMet individuals ( $t=2.03, d f=19.32, P=.06$ ), whereas no difference was observed between ValMet and MetMet individuals $(P>31)$ (see Figure 2). This interaction remained statistically significant after adjustment for age $(P=.032)$ and was reduced to a strong trend after adjustment for illness duration $(P=.051)$. Notably, the lower dIPFC activity in ValVal individuals during high-load specific WM was also observed in post hoc comparison of ValVal individuals with all Met carriers (MetMet and ValMet) $(t=2.81, d f=62, P=.007$ ). No significant effects of COMT genotype and gender were found on high-load specific dIPFC activity $(P>.99)$.

\subsection{2 | Whole-brain exploratory analyses}

High-load specific (2-back>1-back) WM activated a widespread frontoparietal network of overlapping regions, including the right and left dIPFC (for peak cluster activations, see Table 3), consistent with the literature. ${ }^{45}$ Exploratory whole-brain analyses revealed no effect of genotype for either high-load specific or general WM ( $F$ tests assessing possible differences between genotype groups). Prompted by our ROI finding, we also tested whether the differential right dIPFC activity in ValVal vs ValMet individuals during high-load specific WM extended to additional brain regions. This whole-brain analysis revealed four clusters within the left and right dIPFC showing reduced high-load specific activity in ValVal compared with ValMet individuals in all clusters (with MetMet individuals consistently showing intermediate responses; see Figure 3).

\subsection{Associations between mood, medication, BOLD response and behavior}

Across the entire sample, subsyndromal mania and depression symptoms showed no correlation with high-load specific dIPFC activity $(P \geq 28)$ or performance on the $n$-back WM task during the scan $(P \geq .35)$. There was also no association between mood symptoms and SWM strategy $(P \geq 14)$, although more subsyndromal depressive symptoms correlated weakly with more SWM errors $(r=.26, P=.04)$. The use of lithium, anticonvulsants, antidepressants, antipsychotics or benzodiazepines was not associated with high-load specific dIPFC activity $(P \geq 14)$ or with performance on the $n$-back and SWM tests across the entire cohort ( $P \geq .21$ and $P \geq .06$, respectively).

There were no interactions between COMT Val158Met genotype and the total number of medications for $n$-back WM performance $(P \geq .38)$ or WM-related dIPFC activity $(P \geq .53)$. There were also no interactive effects of genotype and the number of medications on SWM performance (strategy: $P \geq .33$; errors: $P \geq .46$ ). There was a trend towards correlation between more high-load specific dIPFC activity and better $n$-back WM performance across the entire sample $(r=.21$, $d f=64, P=.10)$ as well as within the Valval group ( $r=.54, d f=13, P=.058$. Post hoc analysis showed that better SWM performance (reflected by lower strategy scores and fewer errors) correlated weakly with better $n$-back WM accuracy across the entire sample (SWM strategy: $r=-.27$, $d f=59, P=.002$; SWM errors: $r=-.40, d f=59, P=.002$ ).

\section{4 | DISCUSSION}

The present fMRI study examined the effects of the functional COMT Val158Met polymorphism on WM-related neural activity in partially or fully remitted BD patients. Based on previous studies of schizophrenia and healthy groups, ${ }^{37}$ we had hypothesized that Val homozygous BD patients would exhibit increased activity in the dIPFC during high-load WM relative to Met carriers. Contrary to our predictions, Val homozygous patients showed decreased dIPFC activity during high-load WM compared with Met carriers. We observed no effect of genotype on n-back WM performance during the scan. However, as hypothesized, Val carriers displayed poorer performance than Met homozygotes on the CANTAB SWM task outside the scanner, as reflected by less efficient strategies and more errors (effect sizes: Cohen's $d=0.98-1.90$ ). The observed effects of genotype on WM-related neural activity and spatial WM performance in our BD cohort occurred in the absence of differences between genotypes in demographic characteristics, subsyndromal mood symptoms or medications.

The relatively reduced dIPFC activity during high-load specific WM in Val homozygous patients was unexpected given evidence pointing to hyperactivity and purported inefficient dIPFC function in Val carriers. ${ }^{13,30-35}$ However, it is conceivable that Val homozygous patients' lower dIPFC activity reflects failure to recruit task-relevant dIPFC resources, given their impaired performance on the CANTAB SWM task. Indeed, decreased right dIPFC activity during high WM loads in Val homozygous patients with BD is consistent with the literature on 


\begin{tabular}{|c|c|c|c|c|c|}
\hline \multirow[b]{2}{*}{ Regional response } & \multirow{2}{*}{$\begin{array}{l}\text { Cluster size } \\
\text { (no. voxels) }\end{array}$} & \multirow{2}{*}{$\begin{array}{l}\text { Cluster } \\
\text { P-value }\end{array}$} & \multicolumn{3}{|c|}{ Coordinates } \\
\hline & & & $x$ & $y$ & $z$ \\
\hline \multicolumn{6}{|c|}{ High-load specific WM (2-back>1-back) across the entire cohort (main effect of task) } \\
\hline Frontal cortex & 21675 & .001 & & & \\
\hline Left superior frontal gyrus (BA6) & & & -20 & 0 & 74 \\
\hline Right middle frontal gyrus (BA6) & & & 46 & 20 & 46 \\
\hline Left middle frontal cortex (BA32) & & & -2 & 38 & 25 \\
\hline Right thalamus & & & 18 & -22 & 18 \\
\hline Left middle frontal gyrus (BA9) & & & -50 & 26 & 32 \\
\hline Parietal cortex & 18079 & .001 & & & \\
\hline Left precuneus (BA7) & & & -4 & -62 & 56 \\
\hline Right supramarginal gyrus (BA40) & & & 56 & -36 & 42 \\
\hline Left supramarginal gyrus (BA40) & & & -48 & -40 & 38 \\
\hline Right lateral occipital cortex (BA18) & & & -34 & -86 & 0 \\
\hline Left lateral occipital cortex (BA19) & & & 38 & -76 & 0 \\
\hline Right cerebellum & 521 & .004 & 32 & -64 & -26 \\
\hline Left cerebellum & 505 & .005 & -32 & -60 & -26 \\
\hline Right inferior temporal gyrus (BA20) & 44 & .036 & 58 & -42 & -14 \\
\hline \multicolumn{6}{|l|}{ ValMet>ValVal (2-back>1-back) } \\
\hline Right superior frontal gyrus (BA6) & 63 & .035 & 22 & 10 & 50 \\
\hline Left middle frontal gyrus (BA8) & 47 & .043 & -46 & 10 & 50 \\
\hline Left middle frontal gyrus (BA6) & 10 & .044 & -36 & -4 & 60 \\
\hline Right superior frontal gyrus (BA8) & 2 & .048 & 36 & 28 & 52 \\
\hline
\end{tabular}

TABLE 3 Peak cluster activation in regions identified in whole-brain analysis as showing decreased response during high-load specific working memory (WM) (2-back>1-back) in catechol-Omethyltransferase (COMT) 158Val homozygotes relative to ValMet individuals

BA, Brodmann area. MNI coordinates $(x, y, z)$ refer to the point of peak activation within each cluster.

the neural underpinnings of cognitive deficits in $\mathrm{BD}$, where the most consistent finding is dIPFC hypoactivity during high-load WM conditions. ${ }^{56,57}$ These findings point to a distinct effect of the COMT genotype on neural activity and cognitive function in patients with $\mathrm{BD}$ as compared to healthy individuals and patients with schizophrenia. Notably, this association between COMT genotype and dIPFC activity was rendered non-significant in a post hoc analysis adjusted for illness duration $(P=.051)$. While the sample size is small, this could suggest that presumed progressive cognitive decline with more chronic illness weakens the association between COMT genotype and dIPFC function in BD.

The demonstration of no behavioral difference between COMT genotype groups on the n-back WM task is in line with previous research utilizing this test in psychiatric and healthy populations. ${ }^{27-29}$ This finding is likely to reflect a ceiling effect attributable to high nback WM accuracy in all participants (i.e., 89\% accuracy across all genotype groups) given (i) the significant correlation between performance on the n-back WM and SWM tests, indicating that the two tests measure the same construct (i.e., spatial WM) and (ii) the separation between groups on performance on the (more difficult) CANTAB SWM test outside the scanner. Indeed, prior evidence suggests that accuracy is generally high in the $n$-back paradigm and that WM loads - even when including three items - fail to yield differences in performance between the COMT Val158Met genotypes. ${ }^{27}$ Nevertheless, the n-back task is arguably the most commonly used fMRI paradigm in the assessment of neural underpinnings of executive function and is optimized for delineating task-relevant neural activity precisely because it ensures high performance accuracy (i.e., neural activity subserving correct performance). The task should thus be combined with more demanding WM tasks devoid of ceiling effects outside the scanner to optimize sensitivity for differences in behavioral read-outs.

Previous research in patients with schizophrenia and healthy individuals has shown that Val homozygotes exhibit exaggerated WMrelated activity in the dIPFC in the absence of behavioral differences. Increased PFC activity has therefore been hypothesized to indicate reduced neural efficiency. ${ }^{27}$ However, the decreased dIPFC activity in Val homozygous BD patients may also be indicative of less efficient recruitment of WM-related neural resources, which could explain these individuals' poorer performance on the SWM test outside the scanner. Indeed, task-related dIPFC hypoactivity in BD has been hypothesized to play a key role in patients' common deficits in attention control and decision making. ${ }^{58}$ Accordingly, both dIPFC hypo- and hyperactivity as observed in ValVal and ValMet individuals, respectively, may reflect PFC dysfunction (deficient recruitment of PFC resources and inefficient processing, respectively), whereas the medium levels of WM-related dIPFC activity in the cognitively superior Met homozygotes may reflect optimal PFC function (see illustration in Figure 4). The reduced PFC function and WM performance in Val carriers (which was most pronounced in Val homozygotes) could be explained by differences in dIPFC cellular pathology in BD relative to 


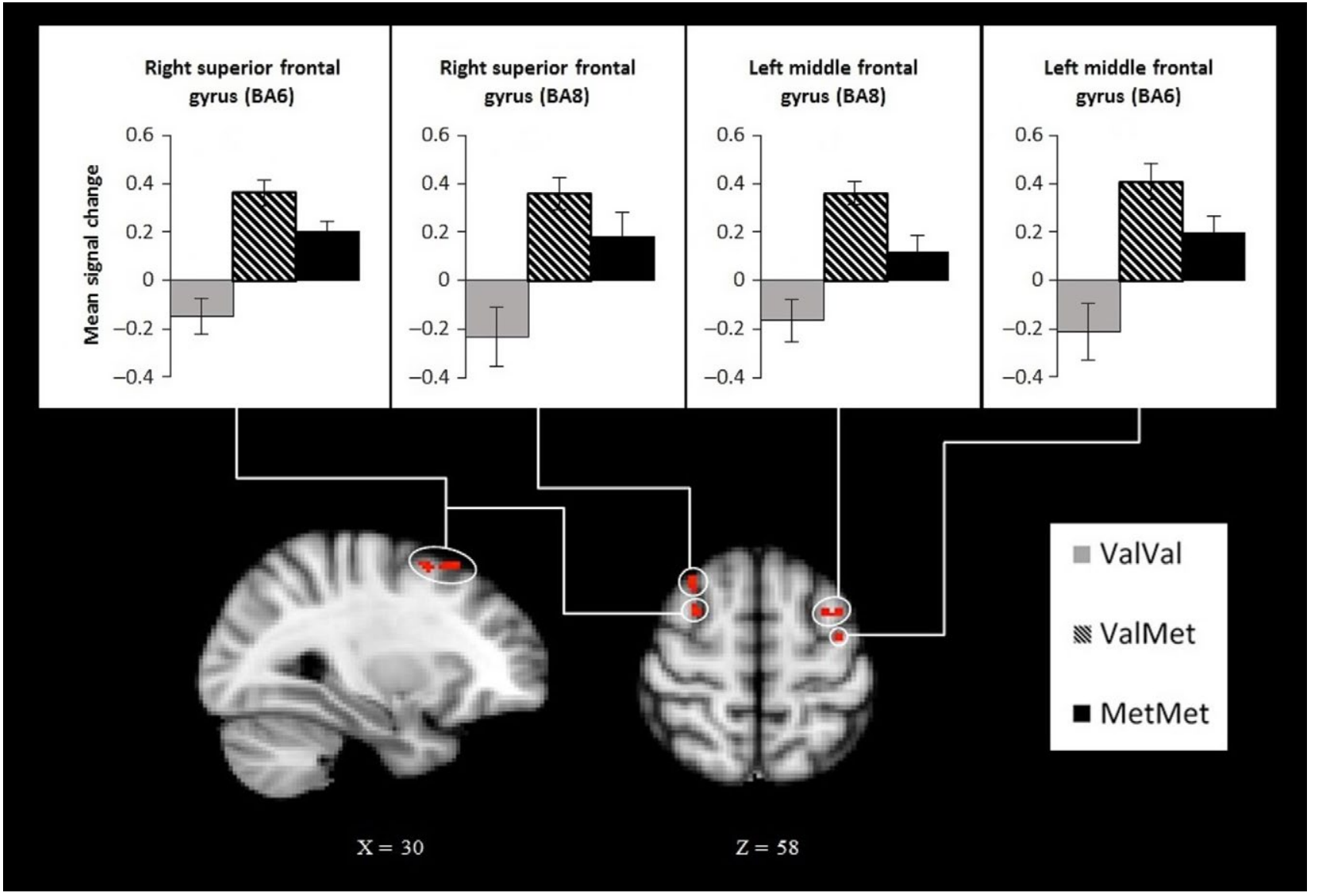

FIGURE 3 Lower part: Neural networks activated during high-load specific working memory (WM) (2-back $>1$-back) across all patients stratified for the catechol-O-methyltransferase (COMT) Val158Met genotype. Clusters show decreased response in ValVal vs ValMet subjects in the right and left dorsolateral prefrontal cortex in the exploratory whole-brain analysis. Data were modeled using a general linear model with nonparametric permutation inference $(n=5000)$, and significant clusters were identified using the threshold-free cluster enhancement method at corrected $P<.05$. Upper part: Bar charts represent mean blood-oxygen-level-dependent (BOLD) signal change during $n$-back high-load specific WM. Bars show the mean; error bars represent the standard error of the mean. [Colour figure can be viewed at wileyonlinelibrary.com]

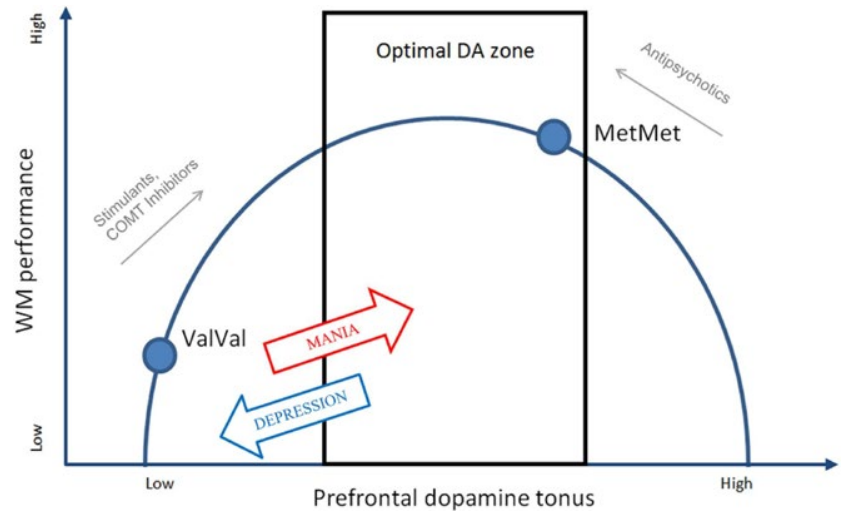

FIGURE 4 Hypothetical inverted-U-shaped interaction between catechol-O-methyltransferase (COMT) Val158Met genotype and the effects of dopaminergic drugs on prefrontal cortex (PFC)-dependent working memory (WM) function in remitted patients with bipolar disorder (BD) (adapted from 22) [Colour figure can be viewed at wileyonlinelibrary.com]

healthy individuals and patients with schizophrenia. ${ }^{59}$ In particular, it is possible that a shift in DA tonus would lead to suboptimal prefrontal DA and greater cognitive impairment in Val homozygotes and (albeit to a lesser degree) in ValMet individuals than in Met homozygous BD patients during remission. Consistent with this, one study has shown that Val carriers exhibit better cognitive performance during manic episodes, presumably due to increased prefrontal DA. ${ }^{38}$ Interestingly, a study investigating the effect of DA agonism on cognitive impairment in clinically stable BD patients found a phase effect of pramipexole, in which only euthymic BD patients showed improvements on a WM task. ${ }^{60}$ It is therefore conceivable that cognitive benefits of increasing prefrontal DA tonus with pramipexole could be specific for patients with relatively low baseline DA, such as patients who are euthymic. Hence, future studies should investigate whether pramipexole treatment may be associated with particularly beneficial cognitive effects in euthymic patients with BD and further investigate the effect of COMT Val158Met genotype on treatment efficacy on cognition.

There is emerging evidence for Val158Met genotype moderation of antipsychotic effects on WM function. Specifically, preclinical evidence suggests that stimulants and other agents that increase prefrontal DA (e.g., methylphenidate and tolcapone) improve WM abilities only in conditions where PFC DA levels are challenged (such as in Val carriers). ${ }^{61-63}$ In contrast, antipsychotics that decrease DA levels (e.g., 
olanzapine) have particularly negative effects on cognition in Val homozygotes ${ }^{64}$ and may be beneficial for Met homozygotes. In keeping with this, it was concluded in a recent systematic review that COMT inhibitors (which lead to DA increase) have the greatest cognitive benefits in Val homozygotes, and that antipsychotics are generally associated with better cognitive outcome among Met homozygotes. ${ }^{22}$ However, this seems to be caused by state-related changes in DA since Val carriers in hyper-dopaminergic manic states exhibit superior cognitive abilities relative to Met carriers. ${ }^{38}$ Hence, in manic states, stimulating DA would theoretically be counterproductive, leading to excessive prefrontal DA and thus cognitive decline. Taken together, the findings suggest that future treatment strategies targeting cognition are likely to benefit from taking patients' COMT Val158Met genotype into consideration. This could provide a basis for studies investigating personalized medicine strategies in BD, in which pharmacological interventions that increase cortical dopaminergic activity (e.g., COMT inhibitors) may be targeted to Val carriers and antipsychotic medications preferentially used in Met homozygotes. ${ }^{22}$

The moderate sample size of the present study may have influenced the validity of the findings, limits generalization of results and impedes further analysis of the effects of medication status on WM abilities by COMT genotype group. The findings therefore require replication in a larger sample. It was also a limitation that we had not recorded information about patients' particular antipsychotic drugs or doses or whether they had experienced psychotic episodes in the course of their illness, as these variables may differ between the COMT Val158Met genotypes and thus potentially confound the findings. Indeed, numerically (but not statistically significantly) more Val homozygotes were treated with antipsychotic drugs. Nevertheless, post hoc analyses revealed no association between antipsychotic treatment and high-load specific dIPFC activity or WM performance across the entire cohort. In addition, the lack of a healthy control group hampered direct assessment of whether the dIPFC in BD Val homozygotes was hypoactive during high-load WM. Another limitation was that the SWM was not administered on the same day as the fMRI scan but merely within the same week and that daily variations in mood were therefore not controlled for Although other single nucleotide polymorphisms (SNPs) have been shown to have an effect on DA availability, cognition, and risk for BD (e.g., rs165599), participants in the present study were stratified only for COMT Val158Met genotype. Nevertheless, the present study is the first to demonstrate the moderating effect of COMT Val158Met genotype on WM-related dIPFC activity and WM performance in a well-characterized partially or fully remitted sample of BD patients.

In conclusion, this is the first study to demonstrate a moderating effect of COMT Val158Met genotype on WM-related dIPFC activity and WM performance in remitted patients with BD. Specifically, Val homozygotes showed less recruitment of task-relevant dIPFC resources during high-load WM and impaired WM performance relative to Met carriers. This is consistent with an association between $\mathrm{Val}$ allelic load and WM impairment in BD and points to a putative role of aberrant PFC dopamine tonus in the cognitive impairments in $\mathrm{BD}$.

\section{ACKNOWLEDGEMENTS}

The study was based on two original trials funded by the TrygFonden, Danish Council for Independent Research, Novo Nordisk Foundation, Beckett Fonden, and Savværksejer Juhl's Mindefond. The study was financed through grant R219-2016-1030 from the Lundbeck Foundation (to L.K.H.). The sponsors had no role in the planning or conduct of the study or in the interpretation of the results.

\section{DISCLOSURES}

K.W.M. reports having received consultancy fees from Lundbeck and Allergan within the past 3 years. M.V. discloses consultancy fees from Lundbeck and Astra Zeneca within the last 3 years. H.R.S. discloses honoraria as reviewing editor for Neuroimage, as speaker for Biogen Idec Denmark $A / S$, and as scientific advisor for Lundbeck within the past 3 years. L.V.K. reports having been a consultant for Lundbeck, AstraZeneca and Sunovion within the last 3 years. K.E.B. has served on advisory boards for DSP Pharma, Takeda-Lundbeck, and Neuralstem. T.W. has served as a lecturer for and consultant to $H$. Lundbeck $A / S$. All other authors report no biomedical financial interests or potential conflicts of interest.

\section{REFERENCES}

1. Bourne C, Aydemir O, Balanza-Martinez V, et al. Neuropsychological testing of cognitive impairment in euthymic bipolar disorder: an individual patient data meta-analysis. Acta Psychiatr Scand. 2013;128:149-162.

2. Depp CA, Mausbach BT, Harmell AL, et al. Meta-analysis of the association between cognitive abilities and everyday functioning in bipolar disorder. Bipolar Disord. 2012;14:217-226.

3. Bora E, Yucel M, Pantelis C. Cognitive endophenotypes of bipolar disorder: a meta-analysis of neuropsychological deficits in euthymic patients and their first-degree relatives. J Affect Disord. 2009;113:120.

4. Fernandez-Corcuera P, Salvador R, Monte GC, et al. Bipolar depressed patients show both failure to activate and failure to de-activate during performance of a working memory task. J Affect Disord. 2013;148:170-178.

5. Frangou S, Kington J, Raymont V, Shergill SS. Examining ventral and dorsal prefrontal function in bipolar disorder: a functional magnetic resonance imaging study. Eur Psychiatry. 2008;23:300-308.

6. Hamilton LS, Altshuler LL, Townsend J, et al. Alterations in functional activation in euthymic bipolar disorder and schizophrenia during a working memory task. Hum Brain Mapp. 2009;30:3958-3969.

7. Minzenberg MJ, Laird AR, Thelen S, Carter CS, Glahn DC. MEtaanalysis of 41 functional neuroimaging studies of executive function in schizophrenia. Arch Gen Psychiatry. 2009;66:811-822.

8. Monks PJ, Thompson JM, Bullmore ET, et al. A functional MRI study of working memory task in euthymic bipolar disorder: evidence for task-specific dysfunction. Bipolar Disord. 2004;6:550-564.

9. Townsend J, Bookheimer SY, Foland-Ross LC, Sugar CA, Altshuler LL. fMRI abnormalities in dorsolateral prefrontal cortex during a working memory task in manic, euthymic and depressed bipolar subjects. Psychiatry Res. 2010;182:22-29.

10. Thermenos HW, Goldstein JM, Milanovic SM, et al. An fMRI study of working memory in persons with bipolar disorder or at genetic risk for bipolar disorder. Am J Med Genet B Neuropsychiatr Genet. 2010;153B: 120-131. 
11. Drapier D, Surguladze S, Marshall N, et al. Genetic liability for bipolar disorder is characterized by excess frontal activation in response to a working memory task. Biol Psychiat. 2008;64:513-520.

12. Tunbridge EM, Harrison PJ, Weinberger DR. Catechol-omethyltransferase, cognition, and psychosis: Val 158 Met and beyond. Biol Psychiat. 2006;60:141-151.

13. Egan MF, Goldberg TE, Kolachana BS, et al. Effect of COMT $\mathrm{Val}(108 / 158)$ Met genotype on frontal lobe function and risk for schizophrenia. Proc Natl Acad Sci USA. 2001;98:6917-6922.

14. Berk M, Dodd S, Kauer-Sant'Anna M, et al. Dopamine dysregulation syndrome: implications for a dopamine hypothesis of bipolar disorder. Acta Psychiatr Scand. 2007;116:41-49.

15. Pathak G, Ibrahim BA, McCarthy SA, Baker K, Kelly MP. Amphetamine sensitization in mice is sufficient to produce both manic- and depressive-related behaviors as well as changes in the functional connectivity of corticolimbic structures. Neuropharmacology. 2015;95:434-447.

16. Lambert G, Johansson M, Ågren H, Friberg P. Reduced brain norepinephrine and dopamine release in treatment-refractory depressive illness: evidence in support of the catecholamine hypothesis of mood disorders. Arch Gen Psychiatry. 2000;57:787-793.

17. Bressan RA, Costa DC, Jones HM, Ell PJ, Pilowsky LS. Typical antipsychotic drugs - D2 receptor occupancy and depressive symptoms in schizophrenia. Schizophr Res. 2002;56:31-36.

18. Verhoeff NPLG, Christensen BK, Hussey D, et al. Effects of catecholamine depletion on D2 receptor binding, mood, and attentiveness in humans: a replication study. Pharmacol Biochem Behav. 2003;74:425-432.

19. Zarate CA Jr, Payne JL, Singh J, et al. Pramipexole for bipolar II depression: a placebo-controlled proof of concept study. Biol Psychiat. 2004;56:54-60.

20. Martinez-Aran A, Vieta E. Cognition as a target in schizophrenia, bipolar disorder and depression. Eur Neuropsychopharmacol. 2015;25:151-157.

21. Miskowiak K, Ott C, Petersen J, Kessing L. Systematic review of randomized controlled trials of candidate treatments for cognitive impairment in depression and methodological challenges in the field. Eur Neuropsychopharmacol. 2016;26:1845-1867.

22. Schacht J. COMT val158met moderation of dopaminergic drug effects on cognitive function: a critical review. Pharmacogenomics J. 2016;16:430-438

23. Aguilera $M$, Barrantes-Vidal N, Arias $B$, et al. Putative role of the COMT gene polymorphism (Val158Met) on verbal working memory functioning in a healthy population. Am J Med Genet B Neuropsychiat Genet. 2008;147:898-902.

24. Leung WW, McClure MM, Siever LJ, Barch DM, Harvey PD. CatecholO-methyltransferase Val158Met genotype in healthy and personality disorder individuals: preliminary results from an examination of cognitive tests hypothetically differentially sensitive to dopamine functions. Neuropsychiatr Dis Treat. 2007;2007:925.

25. Papenberg G, Bäckman L, Nagel IE, et al. COMT polymorphism and memory dedifferentiation in old age. Psychol Aging. 2014;29:374

26. Sundermann EE, Bishop JR, Rubin LH, et al. Genetic predictor of working memory and prefrontal function in women with HIV. J Neurovirol. 2015;21:81-91.

27. Ihne JL, Gallagher NM, Sullivan M, Callicott JH, Green AE. Is less really more: does a prefrontal efficiency genotype actually confer better performance when working memory becomes difficult? Cortex. 2016;74:79-95.

28. Wardle MC, de Wit H, Penton-Voak I, Lewis G, Munafò MR. Lack of association between COMT and working memory in a populationbased cohort of healthy young adults. Neuropsychopharmacology. 2013;38:1253-1263.

29. Blanchard MM, Chamberlain SR, Roiser J, Robbins TW, Müller U. Effects of two dopamine-modulating genes (DAT1 9/10 and COMT
$\mathrm{Val} / \mathrm{Met})$ on n-back working memory performance in healthy volunteers. Psychol Med. 2011;41:611-618.

30. Bertolino A, Blasi G, Latorre V, et al. Additive effects of genetic variation in dopamine regulating genes on working memory cortical activity in human brain. J Neurosci. 2006;26:3918-3922.

31. Bertolino A, Di Giorgio A, Blasi G, et al. Epistasis between dopamine regulating genes identifies a nonlinear response of the human hippocampus during memory tasks. Biol Psychiatry. 2008;64:226-234.

32. Caldú $X$, Vendrell $P$, Bartrés-Faz $D$, et al. Impact of the COMT Val108/158 Met and DAT genotypes on prefrontal function in healthy subjects. Neurolmage. 2007;37:1437-1444.

33. Meyer-Lindenberg A, Nichols T, Callicott JH, et al. Impact of complex genetic variation in COMT on human brain function. Mol Psychiatry. 2006:11:867-877.

34. Tan $\mathrm{H}-\mathrm{Y}$, Chen Q, Sust $\mathrm{S}$, et al. Epistasis between catechol-Omethyltransferase and type II metabotropic glutamate receptor 3 genes on working memory brain function. Proc Natl Acad Sci USA. 2007:104:12536-12541.

35. de Frias CM, Marklund P, Eriksson E, et al. Influence of COMT gene polymorphism on $\mathrm{fMRI}$-assessed sustained and transient activity during a working memory task. J Cogn Neurosci. 2009;22:1614-1622.

36. Wheaton MG, Fitzgerald DA, Phan KL, Klumpp H. Perceptual load modulates anterior cingulate cortex response to threat distractors in generalized social anxiety disorder. Biol Psychol. 2014;101:13-17.

37. Mier D, Kirsch P, Meyer-Lindenberg A. Neural substrates of pleiotropic action of genetic variation in COMT: a meta-analysis. Mol Psychiatry. 2010;15:918-927.

38. Soeiro-de-Souza MG, Machado-Vieira R, Soares Bio D, Do Prado CM, Moreno RA. COMT polymorphisms as predictors of cognitive dysfunction during manic and mixed episodes in bipolar I disorder. Bipolar Disord. 2012;14:554-564.

39. Burdick KE, Funke B, Goldberg JF, et al. COMT genotype increases risk for bipolar I disorder and influences neurocognitive performance. Bipolar Disord. 2007;9:370-376.

40. Haas BW, Omura K, Constable RT, Canli T. Emotional conflict and neuroticism: personality-dependent activation in the amygdala and subgenual anterior cingulate. Behav Neurosci. 2007;121:249-256.

41. Flint J, MunafÒ MR. The endophenotype concept in psychiatric genetics. Psychol Med. 2007;37:163-180.

42. Miskowiak KW, Ehrenreich H, Christensen EM, Kessing LV, Vinberg M. Recombinant human erythropoietin to target cognitive dysfunction in bipolar disorder: a double-blind, randomized, placebo-controlled phase 2 trial. J Clin Psychiatry. 2014;75:1347-1355.

43. Demant KM, Vinberg M, Kessing LV, Miskowiak KW. Effects of shortterm cognitive remediation on cognitive dysfunction in partially or fully remitted individuals with bipolar disorder: results of a randomised controlled trial. PLoS ONE. 2015;10:e0127955.

44. Fava M, losifescu DV, Pedrelli P, Baer L. Reliability and validity of the Massachusetts general hospital cognitive and physical functioning questionnaire. Psychother Psychosom. 2009;78:91-97.

45. Wager TD, Smith EE. Neuroimaging studies of working memory: a meta-analysis. Cogn Affect Behav Neurosci. 2003;3:255-274.

46. Miskowiak KW, Vinberg M, Glerup L, et al. Neural correlates of improved executive function following erythropoietin treatment in mood disorders. Psychol Med. 2016;46:1679-1691.

47. Demant KM, Almer GM, Vinberg M, Kessing LV, Miskowiak KW. Effects of cognitive remediation on cognitive dysfunction in partially or fully remitted patients with bipolar disorder: study protocol for a randomized controlled trail. Trials 2013;14:745-6215.

48. Gonzalez-Castillo J, Roopchansingh V, Bandettini PA, Bodurka J. Physiological noise effects on the flip angle selection in BOLD fMRI. Neurolmage. 2011;54:2764-2778.

49. Maldjian JA, Laurienti PJ, Kraft RA, Burdette JH. An automated method for neuroanatomic and cytoarchitectonic atlas-based interrogation of fMRI data sets. Neurolmage. 2003;19:1233-1239. 
50. Woolrich MW, Ripley BD, Brady M, Smith SM. Temporal autocorrelation in univariate linear modeling of FMRI data. Neurolmage. 2001;14:1370-1386.

51. Winkler AM, Ridgway GR, Webster MA, Smith SM, Nichols TE. Permutation inference for the general linear model. Neurolmage. 2014;92:381-397.

52. Talairach J, Tournoux P. Co-Planar Stereotaxic Atlas of the Human Brain. 3-Dimensional Proportional System: An Approach to Cerebral Imaging. New York: Thieme; 1988.

53. Grier JB. Nonparametric indexes for sensitivity and bias: computing formulas. Psychol Bull. 1971;75:424.

54. Tunbridge EM, Harrison PJ. Importance of the COMT gene for sex differences in brain function and predisposition to psychiatric disorders. In: Neill JC, Kulkarni J, eds. Biological Basis of Sex Differences in Psychopharmacology. Springer, Berlin Heidelberg: Berlin, Heidelberg; 2011:119-140.

55. McDonald J. Handbook of Biological Statistics. Baltimore, MD: Sparky House Publishing; 2014.

56. Lim CS, Baldessarini RJ, Vieta E, Yucel M, Bora E, Sim K. Longitudinal neuroimaging and neuropsychological changes in bipolar disorder patients: review of the evidence. Neurosci Biobehav Rev. 2013;37:418-435.

57. Pomarol-Clotet E, Alonso-Lana S, Moro N, et al. Brain functional changes across the different phases of bipolar disorder. Br J Psychiat. 2015;206:136-144.

58. Malhi GS, Byrow Y, Fritz K, et al. Mood disorders: neurocognitive models. Bipolar Disord. 2015;17:3-20.

59. Rajkowska G, Halaris A, Selemon LD. Reductions in neuronal and glial density characterize the dorsolateral prefrontal cortex in bipolar disorder. Biol Psychiat. 2001;49:741-752.
60. Burdick KE, Braga RJ, Nnadi CU, Shaya Y, Stearns WH, Malhotra AK. A placebo-controlled adjunctive trial of pramipexole in patients with bipolar disorder: targeting cognitive dysfunction. J Clin Psychiatry. 2012;73:103-112.

61. Giakoumaki SG, Roussos P, Bitsios P. Improvement of prepulse inhibition and executive function by the COMT inhibitor tolcapone depends on COMT Val158Met polymorphism. Neuropsychopharmacology. 2008;33:3058-3068.

62. Mattay VS, Goldberg TE, Fera F, et al. Catechol O-methyltransferase val158-met genotype and individual variation in the brain response to amphetamine. Proc Natl Acad Sci. 2003;100:6186-6191.

63. Farrell SM, Tunbridge EM, Braeutigam S, Harrison PJ. COMT Val 158 Met genotype determines the direction of cognitive effects produced by catechol-O-methyltransferase inhibition. Biol Psychiat. 2012;71:538-544.

64. Arts B, Simons CJ, Drukker M, van Os J. Antipsychotic medications and cognitive functioning in bipolar disorder: moderating effects of COMT Val 108/158 Met genotype. BMC Psychiatry. 2013;13:1.

How to cite this article: Miskowiak KW, Kjærstad $\mathrm{HL}$, Støttrup MM, et al. The catechol-O-methyltransferase (COMT) Val158Met genotype modulates working memory-related dorsolateral prefrontal response and performance in bipolar disorder. Bipolar Disord. 2017;19:214224. https://doi.org/10.1111/bdi.12497 